\title{
Efectividad de la terapia miofuncional en la parálisis facial de tipo periférica y central en la población infantil y adulta.
}

\section{Effectiveness of myofunctional therapy in the facial paralysis of peripheral and central type in the child and adult population.}

Jaimes-rojas, Maryory-Yulixa ${ }^{1}$, Peralta-Silva, Angie-Carolina ${ }^{2}$, Rodríguez-Guevara, CristianFelipe $^{3}$, Ramírez-Perdomo, Angelly-Johana ${ }^{4}$

Como citar este artículo: Jaimes-rojas, Maryory-Yulixa; Peralta-Silva, Angie-Carolina; Rodríguez-Guevara, Cristian-Felipe; Ramírez-Perdomo, Angelly-Johana. Efectividad de la terapia miofuncional en la parálisis facial de tipo periférica y central en la población infantil y adulta. Revistas Científica Signos Fónicos. 2020: 6 (1). 24-42.

Recibido: diciembre 10, 2019 Aprobado: marzo 17, 2020.

\section{RESUMEN}

INTRODUCCIÓN: la terapia miofuncional parte como una opción original e innovadora de tratamiento, no ocupante, y de fácil adaptación, por lo que surge la pregunta de la efectividad de esta terapia y sus ejercicios los cuales han permitido la evolución del paciente. MÉTODOS: se llevó a cabo una revisión sistemática de literatura que recopila varios estudios científicos permitiendo generar hipótesis, respondiendo a unas preguntas mediante la revisión sistemática Cochrane, la organización de estas preguntas siguió el marco de preguntas PICO, mediante la selección de artículos con la metodología PRISMA. RESULTADOS: Esta revisión permitió evidenciar la efectividad de la terapia miofuncional en pacientes con parálisis facial; los artículos fueron sometidos a selección de screeming e inclusión siguiendo el diafragma de PRISMA, evidenciando 180 artículos de los cuales se descartaron 60 por no presentar los requisitos de inclusión plasmados en la investigación. ANÁLISIS Y DISCUSIÓN: En el análisis de datos se evidenció que la terapia miofuncional ofrece una alternativa de tratamiento en los casos de parálisis facial mediantes ejercicios de estimulación que brindan una evolución adecuada en los pacientes. CONCLUSIONES: las investigaciones aportan la efectividad de los ejercicios miofuncionales como un tratamiento de fácil acceso y eficaz.

PALABRAS ClAVE Parálisis facial periférica, Parálisis facial central, Terapia miofuncional, ejercicios miofuncionales.

\footnotetext{
${ }^{1}$ Fonoadiologa, Especialización en Gerencia en Servicios de Salud, maryo_jaimes@hotmail.com, Asilo San Jose- Pamplona, Colombia.

${ }^{2,3,4}$ Practicantes.
} 


\begin{abstract}
INTRODUCTION: Myofunctional therapy starts as an original and innovative treatment option, nonoccupying, and easy to adapt, so the question arises of the effectiveness of this therapy and its exercises which have allowed the evolution of the patient. METHODS: A systematic review of the literature that collects several scientific studies that generate hypotheses was carried out, answering some questions through the Cochrane systematic review, the organization of these questions that follow the PICO questions framework, by selecting articles with the PRISMA methodology RESULTS: This review allowed to demonstrate the effectiveness of myofunctional therapy in patients with facial paralysis; The articles were screened and included for selection following the PRISMA diaphragm, showing 180 articles, of which 60 were discarded for not presenting the inclusion requirements embodied in the investigation. ANALYSIS AND DISCUSSION: The data analysis shows that myofunctional therapy offers an alternative treatment in cases of facial paralysis through stimulation exercises that provide adequate evolution in patients. CONCLUSIONS: Research provides the efficacy of myofunctional exercises as an easily accessible and effective treatment.
\end{abstract}

KEYWORDS Peripheral facial paralysis, Central facial paralysis, Myofunctional therapy, myofunctional exercises.

\title{
INTRODUCCIÓN
}

La terapia miofuncional es la encargada de deglución y las demás funciones orofaciales han sido una preocupación de la odontología desde que los cirujanos dentistas iniciaron los tratamientos de reposicionamiento de los dientes (1).

En un tiempo después se considera al dentista como el "Padre de la Motricidad Orofacial" el norteamericano Alfred Rogers, en 1918, fue quien comenzó los estudios, ha profundidad los hábitos musculares, la función de los músculos faciales y así mismo su control facial de estos. En donde Ideo instrumentos mirando la problemática de las personas con dificultades establecido la míoterapia al igual que también propuso principios terapéuticos para las personas que son respiradores orales, reconociendo la importancia del sistema orofacial en problemas de tratamiento ortodóncico.

Rogers recomendó ejercicios para el desarrollo de los músculos de la cara y para aumentar su actividad funcional. Creo ejercicios para los músculos maseteros, temporales, pterigoideo, orbicular de la boca y músculos faciales. En la época, prácticamente mirando los estudios realizados por el mirando su veracidad de los ejercicios aceptaron la mayor parte de los ortodoncistas el método de Rogers (1).

Dando gracias a estas investigaciones del gran ortodoncista Alfred Rogers, el cual se debe a sus estudios de las funciones de los músculos faciales; en Brasil se diversifico y naturalmente paso a tener especialistas por el consejo federal de fonoaudiología por medio de la Resolución 130/95, donde se determina cuatro áreas especializadas de la fonoaudiología brasilera: Motricidad orofacial, Lenguaje, Voz y Audiología (2).

Después de haber descubierto las cuatro áreas, la motricidad orofacial se torna como un estudio de potencia de acuerdo con las publicaciones de libros y artículos científicos ya existentes en su época para encontrar mejores resultados terapéuticos.

Los primeros brasileros que investigaron sobre la terapia míofuncional el cual poseían poca o casi ninguna literatura nacional para avanzar sus investigaciones.

En donde solo dos libros clásicos en el área de Motricidad orofacial, interrelaciones entre la Odontoestagmatologia y fonoaudiología de María Luiza Segovia publicado en 1977 Argentina, y “Oral 
Jaimes, Peralta, Rodríguez, Ramírez. Revista Científica Signos Fónicos, 2020, 6 (2): 24-42.

Myofuncional Disorders", de Richard Barret y Marvin Hanson, publicado en Estados Unidos 1974, estos libros fueron utilizados por las personas que querían profundizar sus conocimientos en la especialidad de Motricidad Orofacial.

Lischer fue quién en 1912, introdujo el término "Terapia Miofuncional", el cual es una disciplina que se encarga de prevenir, valorar, y corregir las disfunciones orofaciales que pueden interferir tanto en la producción del habla como sobre la estructura de los dientes. No obstante, fue Straub el verdadero responsable del inicio de la terapia mío funcional como tratamiento (2) con el fin de lograr un equilibrio anatómico y funcional de estructuras orofaciales, volviendo a integrar los movimientos que se encuentran ausentes, por lo siguiente ayudando a aumentar la tonicidad orofacial, posibilitando un mejor desarrollo y funcionalidad de las estructuras afectadas.

Por lo siguiente la musculatura de la mímica facial se localiza en las capaz subcutánea insertados en la cara del cráneo y la piel, estos músculos tienen la función esfinteriana y dilatadora de las estructuras encargadas por el movimiento de los orificios de la boca, los ojos y de la nariz.

Lo que promueve la funcionalidad del musculo esquelético estriado es el sarcómero, que se compone de filamentos espesos (miosina) y finos (actina, tropomiosina y troponina).

Para desencadenar la contracción de la fibra muscular esquelética necesitamos de un estímulo eléctrico provenientemente de la motoneurona. Este estímulo culmina en la unión neuromuscular, formada por la motoneurona y la célula muscular. En esa zona, modificaciones en el calcio, el sodio la acetilcolina produce la excitación y consecuente contracción de la fibra muscular (2).

Esta investigación surge de la necesidad de mirar la efectividad de la terapia miofuncional en la patología de parálisis fácil periférica y los ejercicios Miofuncionales más efectivos en niños y en adultos dado que en Colombia el 35\% de la población presenta este tipo de patología, aunque normalmente los casos son leves y se recuperan antes de tres semanas siempre y cuando se estimule con terapia miofuncional, dicen los expertos.

Este tipo de intervención se emplea tanto en niños, como en adolescentes y adultos que presentan alguna disfunción en el sistema orofacial.

El tratamiento miofuncional debe ser llevada a cabo por un profesional especializado en Logopedia, que se encargará de prevenir, evaluar, diagnosticar, educar y rehabilitar aquellas alteraciones que comprometan la funcionalidad del sistema orofacial en patologías de diversa índole (físicas, genéticas, neurológicas o adquiridas) en personas de todas las edades (2).

Para resolver esta investigación se plantearon 3 preguntas:

¿Efectividad de la terapia Miofuncional en la parálisis facial de periférica? Siguiendo esta otra pregunta ¿Cuáles son los ejercicios Miofuncionales más efectivos en la parálisis facial de tipo periférica y central en la población infantil ( 0 a 18 años)? y ¿Cuáles son los ejercicios Mio funcionales más efectivos en parálisis central y periféricas en niños ( 0 - 18 años)?

\section{MÉTODOS}

Para esta investigación se realizó una revisión sistemática donde se tiene en cuenta la metodología Cochrane-PRISMA.Una revisión sistemática tiene como objetivo reunir toda la evidencia empírica que cumple unos criterios de elegibilidad previamente establecidos, con el fin de responder una pregunta específica de investigación (3).

Esta revisión se basó en tres preguntas de investigación, el cual busca generar conocimiento de la efectividad de la Terapia Miofuncional, en la rehabilitación de la parálisis facial de tipo central (PFC), Revista Científica Signos Fónicos, 2020, 6 (2): 1-21. ISNN 2422-1716. 
Jaimes, Peralta, Rodríguez, Ramírez. Revista Científica Signos Fónicos, 2020, 6 (2): 24-42. parálisis facial periférica (PFP), Ejercicios de la terapia Miofuncional en la parálisis facial periférica o de tipo central en la población adulta (18 años en adelante).

Para la elaboración de las preguntas se tienen en cuenta el marco de preguntas PICO (4), donde el objetivo de la presente investigación es observar la efectividad de la terapia Miofuncional en parálisis facial de tipo periférica y central. Las preguntas se presentan a continuación Tabla 1, Tabla 2, Tabla 3.

TABLA No 1. Pregunta PICO para la pregunta por la efectividad de la TM en la parálisis facial de tipo periférica.

\begin{tabular}{|c|c|c|c|}
\hline $\mathbf{P}$ & I & $\bar{C}$ & $\bar{O}$ \\
\hline $\begin{array}{l}\text { Paciente, Población o } \\
\text { Problema }\end{array}$ & Intervención & Comparación & Resultado \\
\hline $\begin{array}{l}\text { Efectividad de la terapia } \\
\text { mío funcional en la } \\
\text { parálisis facial de tipo } \\
\text { periférica }\end{array}$ & $\begin{array}{l}\text { Terapia } \\
\text { míofuncional }\end{array}$ & & $\begin{array}{l}\text { Verificar si la terapia } \\
\text { míofuncional fue efectiva en } \\
\text { la parálisis facial de tipo } \\
\text { periférica }\end{array}$ \\
\hline
\end{tabular}

Fuente: Autores.

TABLA No 2. Pregunta PICO para la pregunta efectividad de los ejercicios Mio funcionales en parálisis facial central y periférica.

\begin{tabular}{llll}
\hline $\begin{array}{l}\text { Paciente, Población o } \\
\text { Problema }\end{array}$ & I & C & O \\
\hline $\begin{array}{l}\text { Efectividad de la terapia } \\
\text { míofuncional en la } \\
\text { parálisis facial de tipo }\end{array}$ & Terapia mío funcional & Comparación & Resultado \\
$\begin{array}{l}\text { central } \\
\end{array}$ & & $\begin{array}{l}\text { Verificar si la } \\
\text { terapia } \\
\text { míofuncional fue } \\
\text { efectiva en la } \\
\text { parálisis facial de } \\
\text { tipo central }\end{array}$ \\
\hline
\end{tabular}

Pregunta de investigación: ¿Cuáles son los ejercicios mío funcionales más efectivos en la parálisis facial de tipo periférica y central en la población infantil ( 0 a 18 años)

Fuente: Autores.

Tabla No 3. Preguntas PICO para la pregunta efectividad de los ejercicios Miofuncionales en parálisis facial central y periférica.

\begin{tabular}{llll}
\hline P & I & C & O \\
$\begin{array}{l}\text { Paciente, Población o } \\
\text { Problema }\end{array}$ & Intervención & Comparación & Resultado \\
\hline $\begin{array}{l}\text { Ejercicios } \\
\begin{array}{l}\text { Miofuncionales más } \\
\text { efectivos en la parálisis }\end{array}\end{array}$ & $\begin{array}{l}\text { Ejercicios de terapia } \\
\text { miofuncional. }\end{array}$ & $\begin{array}{l}\text { Efectividad de los } \\
\text { ejercicios } \\
\text { Miofuncionales. }\end{array}$ \\
\hline
\end{tabular}


Jaimes, Peralta, Rodríguez, Ramírez. Revista Científica Signos Fónicos, 2020, 6 (2): 24-42.

\section{facial de tipo periférica 0 central en la población \\ adulta.}

Pregunta de investigación: ¿Cuáles son los ejercidos Miofuncionales más efectivos en la parálisis facial de tipo periférica o central en la población adulta (18 en adelante)?

\section{Fuente: Autores.}

La declaración PRISMA es una guía de publicación de la investigación diseñada para mejorar la integridad del informe de revisiones sistemática y metaanálisis. Desde su publicación en 2009, los autores investigadores de todo el mundo han utilizado la declaración PRISMA para planificar, preparar y publicar sus revisiones sistemáticas y metaanálisis. La difusión e implantación de la declaración PRISMA parece indicar mejoras en la calidad de la publicación de los métodos y resultados de las revisiones sistemáticas y los metaanálisis (5).

De esta manera la organización y selección de los artículos se desarrolló aplicando los criterios de elegibilidad propuestos en la metodología PRISMA la cual se lleva a cabo en cuatro fases a saber:

\section{a. Fase de identificación}

Se realizaron búsquedas primarias en las bases de datos Science Direct, Scielo, GALE, Taylor y francis y Redalyc Dentro de las búsquedas secundarias se realizaron búsquedas en Google Schoolar, BASE.

\section{b. Fase de Screening}

En esta fase se removieron los artículos duplicados

\section{c. Fase de elegibilidad}

Posterior a la lectura del título y resumen de artículos se procedió con la selección de artículos

\section{d. Fase de inclusión}

Se procedió con la lectura de los textos completos para finalizar con los artículos seleccionados.

\section{RESULTADOS}

Los resultados del proceso de revisión sistemática se certificaron atendiendo el diagrama de flujo de PRISMA, la búsqueda para la pregunta de investigación:

¿efectividad de la terapia miofuncional en la parálisis facial de tipo periférica? Se representa en el Diagrama de la Figura 1, en la cual se reporta que la mayoría de los artículos seleccionados se produjeron en la base de datos Scielo, seguido por REDALYC, TAYLOR y FRACIS y con una menor búsqueda en GALE, sin embargo, en la fase de inclusión y exclusión los artículos que respondían de forma más precisa la pregunta se encuentran en la base de datos REDALYC. Para esta pregunta además de las bases de datos primarias, se procedió con búsquedas secundarias haciendo uso de Google Schoolar y BASE. 
Los resultados del proceso de revisión sistemática se certificaron atendiendo el diagrama de flujo de PRISMA, la búsqueda para la pregunta de investigación: ¿Cuáles son los ejercicios Miofuncionales más efectivos en la parálisis facial de tipo periférica y central en la población infantil de 0 a 18 años? Se representa en el Diagrama de flujo de la Figura 2, en la cual se reporta que la mayoría de los artículos seleccionados se produjeron en la base de datos SCIELO seguido por REDALYC, TAYLOR Y FRANCIS y con una menor búsqueda en GALE, sin embargo, en la fase de inclusión e exclusión los artículos que respondían de forma más precisa la pregunta se encuentran en la base de datos SCIELO, Para esta pregunta además de las bases de datos primarias, se procedió con búsquedas secundarias haciendo uso de Google Schoolar.

Los resultados del proceso de revisión sistemática se certificaron atendiendo el Figura 3 Diagrama de flujo de PRISMA, la búsqueda para la pregunta de investigación:

¿Cuáles son los ejercicios Miofuncionales más efectivos en la parálisis facial de tipo periférica y central en la población adulta de 18 en adelante? Se representa en el diagrama de la Figura 3, en la cual se reporta que la mayoría de los artículos seleccionados se produjeron en la base de datos SCIENCE DIRECT seguido por REDALYC, SCIELO y con una menor búsqueda en GALE, sin embargo, en la fase de inclusión e exclusión los artículos que respondían de forma más precisa la pregunta se encuentran en la base de datos SCIENCE DIRECT, Para esta pregunta además de las bases de datos primarias, se procedió con búsquedas secundarias haciendo uso de Google Schoolar.

\begin{tabular}{|c|c|c|c|c|}
\hline \multirow{5}{*}{ 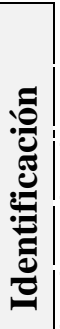 } & Scielo & Redalyc & Taylor y Francis & Gale \\
\hline & $\mathrm{n}=7.320$ & $\mathrm{n}=1.348$ & $\mathrm{n}=132$ & $\mathrm{n}=90$ \\
\hline & \multicolumn{4}{|c|}{ Total, de artículos } \\
\hline & \multicolumn{4}{|c|}{$\mathbf{n}=8,890$} \\
\hline & \multicolumn{3}{|c|}{ Total, de artículos removiendo duplicados } & \\
\hline & \multicolumn{3}{|c|}{$\mathbf{n}=4.820$} & \\
\hline
\end{tabular}

\begin{tabular}{|c|c|c|c|c|}
\hline \multirow{4}{*}{ 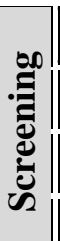 } & \multicolumn{4}{|c|}{ Revisiones bases primarias } \\
\hline & \multicolumn{4}{|c|}{ Artículos seleccionados posterior a lectura de títulos y resúmenes } \\
\hline & Scielo & Redalyc & Taylor y Francis & Gale \\
\hline & $\mathrm{n}=60$ & $\mathrm{n}=35$ & $\mathrm{n}=15$ & $\mathrm{n}=10$ \\
\hline
\end{tabular}

Revista Científica Signos Fónicos, 2020, 6 (2): 1-21. ISNN 2422-1716. 
Jaimes, Peralta, Rodríguez, Ramírez. Revista Científica Signos Fónicos, 2020, 6 (2): 24-42.

\begin{tabular}{|c|c|}
\hline \multicolumn{1}{|c|}{ Revisiones bases secundarias } \\
\hline & Google Schoolar \\
\hline & $\mathbf{n}=455$ \\
\hline & BASE \\
\hline
\end{tabular}

\begin{tabular}{|c|c|c|c|c|}
\hline \multirow{9}{*}{ 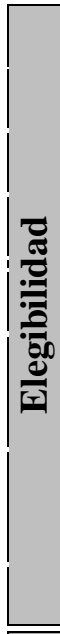 } & \multicolumn{3}{|c|}{ Revisiones bases primarias } & \\
\hline & \multicolumn{3}{|c|}{ Artículos seleccionados posterior a lectura completa } & \\
\hline & Scielo & Redalyc & Taylor y Francis & Gale \\
\hline & $\mathbf{n}=10$ & $\mathbf{n}=8$ & $\mathbf{n}=5$ & $\mathbf{n}=0$ \\
\hline & & & \multicolumn{2}{|c|}{ Tipos de artículos } \\
\hline & & & $\begin{array}{l}\text { 1. Estudios de casos } \\
\text { y controles }\end{array}$ & $\mathbf{n}=7$ \\
\hline & & & \multirow{2}{*}{\multicolumn{2}{|c|}{$\begin{array}{l}\text { 2. Revisiones } \\
\text { sistemáticas }\end{array}$}} \\
\hline & & & & \\
\hline & & & 3. Estudios de caso & $\mathbf{n}=7$ \\
\hline & & & 4. Metaanálisis & $\mathbf{n}=0$ \\
\hline 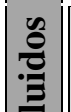 & Total, de artículos incluidos & & $\begin{array}{l}\text { 5. Estudios clínicos } \\
\text { aleatorizados }\end{array}$ & $\mathbf{n}=0$ \\
\hline
\end{tabular}

$$
\mathbf{n}=23
$$

Figura 1. Diagrama de Flujo PRISMA, para la pregunta PICO ¿efectividad de la terapia miofuncional en la parálisis facial de periférica? Fuente: Autores.

\begin{tabular}{|c|c|c|c|c|}
\hline \multirow{7}{*}{$\mid$} & Scielo & Redalyc & Taylor y Francis & Gale \\
\hline & $\mathbf{n}=5.890$ & $\mathbf{n}=2.348$ & $\mathbf{n}=100$ & $\mathbf{n}=80$ \\
\hline & & & & \\
\hline & \multicolumn{4}{|c|}{ Total, de artículos } \\
\hline & \multicolumn{4}{|c|}{$\mathbf{n}=8.418$} \\
\hline & \multicolumn{3}{|c|}{ Total, de artículos removiendo duplicados } & \\
\hline & \multicolumn{3}{|c|}{$\mathbf{n}=3.260$} & \\
\hline
\end{tabular}

\begin{tabular}{|c|c|c|c|c|}
\hline \multirow{5}{*}{ 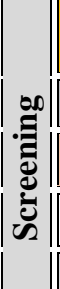 } & \multicolumn{4}{|c|}{ Revisiones bases primarias } \\
\hline & \multicolumn{4}{|c|}{ Artículos seleccionados posterior a lectura de títulos y resúmenes } \\
\hline & Scielo & Redalyc & Taylor y Francis & Gale \\
\hline & $\mathbf{n}=55$ & $\mathbf{n}=35$ & $\mathbf{n}=20$ & $\mathbf{n}=10$ \\
\hline & \multicolumn{4}{|c|}{ Revisiones bases secundarias } \\
\hline
\end{tabular}


Jaimes, Peralta, Rodríguez, Ramírez. Revista Científica Signos Fónicos, 2020, 6 (2): 24-42.

\section{Google Schoolar}

$\mathbf{n}=650$

\begin{tabular}{|c|c|c|c|}
\hline \multicolumn{4}{|c|}{ Revisiones bases primarias } \\
\hline \multicolumn{1}{|c|}{ Artículos seleccionados posterior a lectura completa } \\
\hline Scielo & Redalyc & Taylor y Francis & Gale \\
\hline $\mathbf{n}=8$ & $\mathbf{n}=5$ & $\mathbf{n}=4$ & $\mathbf{n}=1$ \\
\hline
\end{tabular}

\section{Tipos de artículos}

\begin{tabular}{l}
$\begin{array}{l}\text { 1. Estudios de casos } \\
\text { y controles }\end{array}$ \\
\hline
\end{tabular}

2. Revisiones $\quad \mathbf{n}=7$
sistemáticas

3. Estudios de caso $\quad \mathbf{n}=3$

4. Metaanálisis $\quad \mathbf{n}=3$

5. Estudios clínicos $\quad \mathbf{n}=0$
aleatorizados $=0$

Figura 2. Diagrama de Flujo PRISMA, para la pregunta PICO ¿Cuáles son los ejercidos Miofuncionales más efectivos en la parálisis facial de tipo periférica o central en la población infantil (de 0 hasta 18 años)?

\begin{tabular}{|c|c|c|c|c|}
\hline \multirow{6}{*}{ 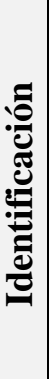 } & Science Direct & Redalyc & Scielo & Gale \\
\hline & $\mathbf{n}=8.348$ & $\mathbf{n}=1.977$ & $\mathbf{n}=132$ & $\mathbf{n}=75$ \\
\hline & \multicolumn{4}{|c|}{ Total, de artículos } \\
\hline & \multicolumn{4}{|c|}{$\mathbf{n}=10.582$} \\
\hline & \multicolumn{3}{|c|}{ Total, de artículos removiendo duplicados } & \\
\hline & \multicolumn{3}{|c|}{$\mathbf{n}=4.260$} & \\
\hline
\end{tabular}

\begin{tabular}{|c|c|c|c|c|}
\hline \multirow{7}{*}{ 串 } & \multicolumn{4}{|c|}{ Revisiones bases primarias } \\
\hline & \multicolumn{4}{|c|}{ Artículos seleccionados posterior a lectura de títulos y resúmenes } \\
\hline & Science Direct & Redalyc & 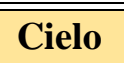 & Gale \\
\hline & $\mathrm{n}=35$ & $\mathrm{n}=25$ & $\mathrm{n}=20$ & $\mathrm{n}=7$ \\
\hline & \multicolumn{4}{|c|}{ Revisiones bases secundarias } \\
\hline & \multicolumn{4}{|c|}{ Google Schoolar } \\
\hline & & $\mathbf{n}=90$ & & \\
\hline
\end{tabular}


Jaimes, Peralta, Rodríguez, Ramírez. Revista Científica Signos Fónicos, 2020, 6 (2): 24-42.

\section{Revisiones bases primarias}

\section{Artículos seleccionados posterior a lectura completa}

\begin{tabular}{|c|c||c||c|}
\hline \hline Science Direct & Redalyc & Cielo & Gale \\
\hline \hline $\mathbf{n}=9$ & $\mathbf{n}=7$ & $\mathbf{n}=3$ & $\mathbf{n}=0$ \\
\hline
\end{tabular}

Tipos de artículos

1. Estudios de casos
y controles

2. Revisiones
sistemáticas

\begin{tabular}{|ll|}
\hline 3. Estudios de caso & $\mathbf{n}=3$ \\
\hline 4. Metaanálisis & $\mathbf{n}=2$ \\
\hline $\begin{array}{l}\text { 5. Estudios clínicos } \\
\text { aleatorizados }\end{array}$ & $\mathbf{n}=0$ \\
\hline
\end{tabular}

$\mathbf{n}=19$

Figura 3. Diagrama de Flujo PRISMA, para la pregunta PICO ¿Cuáles son los ejercidos Miofuncionales más efectivos en la parálisis facial de tipo periférica o central en la población adulta (18 en adelante)?

\section{ANÁLISIS Y DISCUSIÓN}

¿Efectividad de la terapia miofuncional en la parálisis facial de tipo periférica?

La parálisis facial periférica (PFP) se presenta como una debilidad o ausencia de la movilidad de los músculos de un lado de la cara; esto sucede cuando el nervio facial que controla un lado de la cara se daña o se deteriora algún punto de su recorrido y deja de funcionar.

Donde podemos evidenciar que el nervio facial es el que más se lesiona de todos los pares craneales causando el trastorno neuromuscular llamado parálisis facial. Debido a la lesión del nervio facial se impide el movimiento normal de diferentes músculos como: frente, párpados y boca; así como, la expresión de las emociones. Causando la parálisis facial asimetría facial (6), (7), (8).

Las consideraciones para una parálisis facial periferia; es causada por la afección aguda del nervio facial a nivel periférico; originando la pérdida del movimiento voluntario en todos los músculos faciales del lado afectado y es la más común de las parálisis(6),(9),(2), además que la parálisis facial periférica puede ser a nivel unilateral o bilateral, completa si abarca todos los músculos del lado afectado o parcial si solo afecta algunas estructuras orofaciales, la parálisis más común es unilateral, afectando la hemicara ipsilateral del nervio lesionado.

Además, se demuestra que en las sesiones de terapia se produjeron una vez a la semana y la rehabilitación miofuncional discutidos, con la manipulación manual de los músculos de la cara en la dirección de inserción de las fibras musculares, asociado con ejercicios Miofuncionales para la inducción de tono muscular y mejora el movimiento (10), (11). Por lo tanto, ayudando al mejoramiento 
Jaimes, Peralta, Rodríguez, Ramírez. Revista Científica Signos Fónicos, 2020, 6 (2): 24-42.

de la expresión de la mímica y la recuperación de los movimientos fáciles, en el habla masticación, gracias a esto se muestra un favorecimiento en aspectos emocionales y psicológicos.

También se logra evidenciar que la termoterapia en (PFP) tiene como objetivo utilizar el calor para obtener la vascularización de la zona que se está viendo afectada logrando los intercambios celulares en espera del impulso nervioso y en la fase de recuperación de calor y la hipertensión promueve la relajación muscular así mismo regenerando el nervio facial y los músculos de la zona afectada (12), (13), (14).

No obstante, la rehabilitación de los casos de PFP, tradicionalmente, el terapeuta del habla desarrolla una rehabilitación miofuncional de trabajo, a partir de la investigación de los daños causados por los músculos de la lesión del nervio facial. En este enfoque, el profesional evalúa las funciones relacionadas con esta musculatura, intervino con el fin de recuperar los aspectos funcionales y estéticos (15), (16).

Así se muestra una mejoría significativa en musculo esquelética altura orbicular del ojo izquierdo, no hay temblores en la región y la mejora relativa en musculo esquelética altura y la movilidad de la región oral (izquierda) teniendo en cuenta las condiciones establecidas en el comienzo de la rehabilitación miofuncional (15), (17), (18).

Por lo tanto, el paciente muestra un compromiso con los ejercicios Miofuncionales aumento. El paciente mostró una mejoría significativa en el músculo orbicular del ojo izquierdo, no hay temblores en la región y relativa en los músculos y la movilidad de la región oral (izquierda), la sensación también informó de "ligereza" en la cara, teniendo en cuenta las condiciones presentadas anteriormente la rehabilitación miofuncional (15), (19), (20).

De igual modo se observa que el trabajo miofuncional ha demostrado su eficacia, como la etapa flácida, antes de la inervación de los nervios, para ayudar a la recuperación funcional final. Los estímulos realizados en sujetos afectados por la PFP, antes de llegar a los primeros treinta días después de la parálisis instalada, dar mejores resultados que después del período descrito (21), (22).

La parte de rehabilitación de enfoque miofuncional con mioterapia asociada con la sensibilidad propioceptiva y exteroceptivo a la contracción de los músculos paralizados. Los ejercicios ayudan a aumentar el tono de los músculos relajados, de calefacción y de suministro de sangre en la región. En la fase flácida, el ejercicio isométrico por la contracción de los músculos se utiliza en ambos lados de la cara, asociados con masajes, que inducen el lado paralizado (21), (23), (24).

Además de esto la estimulación en los puntos motores faciales conduce a la disminución en el espasmo muscular orofacial, la mejora de la circulación sanguínea y la oxigenación tisular. La estimulación de los músculos orofaciales implica activo y el estiramiento pasivo, que aumentan de sentido percepción de cada músculo en cuestión, la modificación del tono muscular y buscando el equilibrio orofacial miofuncional (21), (25).

La literatura se ha ocupado de la contribución de la terapia del habla en la rehabilitación de la simetría facial. Y el enfoque contextualizado miofuncional orofacial terapia del habla en la mejora de la coordinación y control de ganancia articulatorio bucal durante la masticación y la deglución. El resultado fue satisfactorio, pero no hay necesidad de más publicaciones en miofuncional orofacial y el enfoque de la parálisis facial (26), (27). 
Se lleva a cabo esta investigación, de tipo revisión sistemática para saber la importancia y la necesidad de la Terapia miofuncional en el caso de una parálisis facial periférica, logrando evidenciar la eficacia de la terapia miofuncional en la parálisis facial periférica, de esta manera nos soporta que si es efectiva la terapia miofuncional en la (PFP), dando a conocer las mejoría que los pacientes presentan cuando se exponen a la terapia miofuncional se vuelven a reeducar la zona afectada de la cara, mejorando su movilidad, tonicidad, deglución y articulación.

Así mismo se demuestra que al ver los músculos relajados por causa de la (PFP) al intervenir con la terapia miofuncional en las zonas afectadas vuelve a circular sangre y oxigenación, que le permitirán una mejor movilidad de los músculos faciales, mejorando el aspecto facial de cada individuo, gracias a la terapia miofuncional que implementa sus técnicas para la corrección del desequilibrio muscular, por lo tanto creando nuevos patrones musculares en las zonas afectadas para el mejoramiento de su aspectos fáciles, aspecto psicológicos de las personas.

Es importante llevar a cabo esta investigación ya que la parálisis fácil periférica es un trastorno a nivel motor y sensitivo, comprometiendo la musculatura facial de las personas donde se observa la ausencia de la movilidad de los músculos fáciles, el cual se buscó la efectividad de la terapia miofuncional en la parálisis facial periférica por lo siguiente la terapia miofuncional es eficaz en el mejoramiento de la estética de las personas regenerando el nervios facial y las estructuras afectadas.

La terapia miofuncional en la parálisis facial de tipo central y periférica está dirigido a normalizar la funcionalidad de los movimientos relacionados con el sistema orofacial, en la producción de habla, la masticación y la deglución, así como la adecuación de las funciones neurovegetativas y la expresión individual.

Durante la evaluación miofuncional en personas con parálisis facial de tipo central y periférica se debe tener en cuanta al momento de evaluar los siguientes aspectos: Siempre hacer los ejercicios en frente del espejo, no debe sentir dolor o malestar, todos los ejercicios no deberían ser hecho de una manera simétrica y equilibrada, todos los ejercicios no deberían ser estrictamente controlado con el fin de mantener el movimiento armónico y simétrico, hacer los ejercicios todos los días, tres veces al día, mañana, tarde y noche, tome descansos entre los períodos está descansando y no a la fatiga de los músculos, especial cuidado con los ojos, que llevaba gafas de sol, el uso de microporos en la noche para ayudar a cerrar para completar (15), (28), (29).

¿Cuáles son los ejercicios Miofuncionales más efectivos en la parálisis facial de tipo periférica y central en la población infantil de 0 a 18 años?

Los ejercicios Miofuncionales más efectivos en la parálisis facial de tipo central y periférica en niños encontrados en los artículos mediante la revisión sistemática son:

1. Ejercicios isométricos e isotónicos miofuncional

2. Masoterapia

3. Ejercicios faciales

4. Vendaje neuromuscular

A continuación, se describirán los ejercicios Miofuncionales reportados en la revisión sistemática, lo cual se han encontrado en los artículos citados: 
Jaimes, Peralta, Rodríguez, Ramírez. Revista Científica Signos Fónicos, 2020, 6 (2): 24-42.

Ejercicios isotónicos: los ejercicios isotónicos ayudan a mejorar la movilidad del musculo, lo cual aumentan la oxigenación y el aumento de la amplitud de los movimientos. En el cual se realizan los siguientes ejercicios:

- Vibrar los labios

- Chupar líquidos espesos con una pajilla con aumento de diámetro

-Estallido de los labios sin sorbo

Ejercicios isométricos: Estos ejercicios son los encargados de aumentar la fuerza de los músculos, en el cual ayudan a mantener la contracción. En el cual se realizan los siguientes ejercicios:

- Mascar con un soporte de goma(torniquete)

-Enjuague bucal con agua

-Inflar un globo de goma sin ayuda

Masoterapia: El objetivo principal de la masoterapia o masaje miofuncional reside en una estimulación muscular a nivel sensitivo y motriz para activar el sistema nervioso y producir cambios mediante estímulos mecánicos, ejecutando maniobras, de forma metódica, de estiramientos y compresiones rítmicas (30), (31), (32), en el cual estos masajes se pueden llevar acabo con la presión de las manos o con ayuda de instrumentos de vibración.

Ejercicios faciales: El principal objetivo de los ejercicios faciales reside en promover la simetría y la expresión facial, favoreciendo la alimentación y la comunicación por eso son claves en la rehabilitación logopédica de la PFP (30), (33), (34), en el cual se puede realizar los siguientes ejercicios:

- $\quad$ Ejercicios de masticación

- $\quad$ Ejercicios de vibración

- $\quad$ Ejercicios de succión

Vendaje neuromuscular: El vendaje neuromuscular genera efectos fisiológicos: analgésico, aumento de la circulación linfática y sanguínea, reducción de la fatiga muscular, modulación del tono muscular mejorando la interrelación entre las fascias, la postura y la propiocepción articular (19), (35), (36), por ello, se encuentra indicado en el tratamiento de los dolores musculares, para la reducción de edemas y la facilitación de la respuesta muscular durante el movimiento en niños (16), (37).

Es importante destacar que los ejercicios Miofuncionales tienen el objetivo de acelerar el proceso de regeneración del nervio y también la reanudación de las funciones y los movimientos de los músculos de la mímica cara, La prevención de la atrofia muscular, lo que dañaría el proceso de recuperación. (15), (38), (39).

¿Cuáles son los ejercicios Miofuncionales más efectivos en la parálisis facial de tipo periférica y central en la población adulta (18 en adelante)?

Los ejercicios Miofuncionales más efectivos en la parálisis facial de tipo periférica y central en adultos encontrados en los artículos mediante la revisión sistemática son:

1.Vendaje neuromuscular o kinesiotape.

2.Mioterapia.

3.Terapia mímica y kinesioterapia.

Revista Científica Signos Fónicos, 2020, 6 (2): 1-21. ISNN 2422-1716. 
Jaimes, Peralta, Rodríguez, Ramírez. Revista Científica Signos Fónicos, 2020, 6 (2): 24-42.

4.Calor local.

5.Orofaciales

6.isotónicos.

A continuación, se hará una breve descripción de los ejercicios miofuncionales más efectivos encontrados en los artículos citados.

El Vendaje Neuromuscular o Kinesiotape: es una terapia que se ha convertido en una de las más utilizadas por los fonoaudiólogos para problemas orales, de sensibilidad y de incoordinación a la hora de comer.

Es un método terapéutico poco conocido para el tratamiento de problemas como la parálisis facial periférica, trastornos de habla y deglución. El Método Therapy Taping, toma como fundamento el concepto de estimulación tegumentaria, éste aplicado en parálisis facial genera estímulo cutáneo proporcionando aferencias constantes y duraderas hacia la corteza sensorial primaria, lo que permite una mejor integración del sistema somatosensorial (40), (41).

En el ámbito de la Logopedia su uso está menos extendido y sus beneficios no son tan conocidos. Sin embargo, su utilidad en los tratamientos logopédicos queda cada vez más demostrada. Una evidencia que demuestra la eficacia del kinesiotape en la terapia miofuncional, concretamente en el control del babeo. Tonifica la musculatura facial en casos de debilidad muscular, consigue un adecuado cierre labial y reduce la sialorrea (42), (43), (44). Según autores esta técnica consiste en la aplicación de bandas elásticas en vientres musculares para aumento de la circulación sanguínea, estabilidad, drenaje y estimulación de mecano receptores (45), (46).

Este vendaje se utiliza para brindar sensibilidad, tono y movilidad de las estructuras de la cavidad oral que se encuentran paralizadas con el objetivo de prevenir complicaciones respiratorias y alimenticias facilitando la realización de actividades de la vida diaria.

Se ha identificado como uno de los ejercicios poco usados, pero más efectivos para la rehabilitación y estimulación de músculos paralizados en la parálisis facial, por ende, se podría afirmar con certeza que es un ejercicio miofuncional adecuado, que brinda resultados positivos en los pacientes (47), (48), (49).

Ejercicios de mioterapia: estos son los que ayudan a la tonicidad muscular, permite un adecuado crecimiento y desarrollo del músculo facial, estos ejercicios promueven la corrección de anomalías que favorecen el adecuado desarrollo estomatognático y también ayudan al mejoramiento estético del usuario.

La parte de rehabilitación de un enfoque mioterapia asociada con la sensibilidad propioceptiva y exteroceptiva a la contracción de los músculos paralizados. Los ejercicios ayudan a aumentar el tono de los músculos relajados, calefacción y suministro de sangre en la región. En la fase flácida, se utilizan ejercicios isométricos por la contracción de los músculos de ambos lados de la cara, asociada con masajes, que inducen el lado paralizado (21), (50), (51).

Se pudo evidenciar que estos tipos de ejercicios de mioterapia son los adecuados para la rehabilitación de la parálisis facial periférica en adultos, puesto que permite el aumento del tono muscular y la sensibilidad en aquellas zonas paralizadas, por ende, la terapia miofuncional si es efectiva para estos casos.

Revista Científica Signos Fónicos, 2020, 6 (2): 1-21. ISNN 2422-1716. 
Dentro de los ejercicios de mioterapia encontramos lo que son el uso del hielo, los masajes en los puntos motores de la cara, el espejo de uso asociado con favores de trabajo neuromuscular, ejercicios isotónicos como estrategias de relajación y estiramiento asociados al movimiento y el uso de la termoterapia sobre calor húmedo, los cuales permiten una adecuada mejoría en los pacientes con parálisis facial.

Terapia mímica y kinesioterapia: se utiliza para recuperar el movimiento facial simétrico y para reducir o eliminar los problemas asociados a la parálisis en cuanto al déficit de movimiento.

Consiste en sesiones dirigidas de ejercicios para entrenar movimientos correctos de la cara. Éstos se encuentran directamente relacionados con movimientos de expresiones emocionales faciales cotidianos como arrugar frente, abrir y cerrar los ojos, sonreír, fruncir nariz, levantar el labio superior y apretar los labios, entre otros. Regularmente se emplea un espejo para realizar los ejercicios, con la intención que el paciente se observe y mejore su ejecución (6), (52), (53).

Acompañada de este se han realizado electromiografías con el fin de estimular y reactivar el musculo facial, en cuanto a la evaluación miofuncional se destaca como importante la rehabilitación de aspectos clínicos tales como la motricidad orofacial para tener un adecuado funcionamiento de las cavidades intraorales y extraorales, así mismo, como de las funciones estomatognáticas.

Uno de los ejercicios más adecuados y recomendados son aplicar resistencia solo en movimientos aislados y abrir simétricamente los ojos en la realización de movimientos bucales tales como descubrir los dientes, estriar los labios y soplar hacia fuera, también es de suma importancia realizar ejercicios de estiramiento en las zonas afectadas de tensión y relajación con el fin de estimular al paciente y prepararlo para cuando aparezcan momentos de tensión acompañado de movimientos involuntarios.

Se ha identificado como un ejercicio miofuncional efectivo siempre y cuando haya estimulación de extra-terapia en casa, por ende, es importante realizar una psicoeducación por parte del terapeuta para que así el paciente sea consiente que su recuperación está en constancia de lo que el estimule también en su casa, pues así se pueden obtener mejores resultados.

Calor local: es un ejercicio miofuncional que se realiza mediante una compresa húmeda-caliente por 10 minutos en la hemicara afectada, para mejorar la circulación (54), (42), el cual tiene la ventaja de que es un ejercicio que el paciente puede realizar en casa, este ejercicio actúa sobre los músculos, se realiza por medio de fomentación eléctrica o por bolsa de goma con agua caliente, tiende a satisfacer el resultado de un tratamiento activo, se realiza alrededor del oído y del aguajero estilomastoideo, despreocupándose de lo que pueda causar en el musculo y realizando una semana más o menos.

Es cierto que todas las formas de calor pueden emplearse sin embargo se considera más útiles, sobre todo en un principio, para actuar en profundidad sobre el nervio y los músculos, las ondas cortas que, como ya se mencionó, se realiza desde el primer día en sesiones diarias. También desde el comienzo del tratamiento es útil prescribir, al mismo tiempo que las ondas cortas son la aplicación más simple (55),(56).

Orofaciales: son llamados así porque incluye todos los ejercicios de estimulación de la cavidad oral como de lengua, labios, mejillas, soplo, succión y del paladar. En este caso se utilizaron ejercicios 
Jaimes, Peralta, Rodríguez, Ramírez. Revista Científica Signos Fónicos, 2020, 6 (2): 24-42.

como: inflar las mejillas y esquematizar una sonrisa y luego soltarla.

Es importante también utilizar un diseño de la cara con orientación de la fibra muscular y la dirección de masa orofacial que simula el movimiento deseado, y las guías de como el paciente debe auto estimular. El masaje debe hacerse dos veces al día, antes de las comidas. Los ejercicios que debe realizar son:

-Deslizar los dedos en la frente arriba y abajo.

-Deslizamiento de los dedos en la mejilla desde la parte inferior hacia arriba.

-Deslizar los dedos en los labios, simulando una sonrisa horizontal.

- Uso de la masticación funcional lado paralizado: el apoyo a la base de la mandíbula con los dedos sobre el lado afectado (55) (57), (58).

Es importante resaltar que para conseguir evolución en el paciente por medio de la terapia miofuncional, el paciente debe hacer su propia estimulación en casa, de esta manera pues se verán notorios resultados, sin embargo, se puede decir que estos ejercicios si son efectivos para la rehabilitación de la parálisis facial en adultos.

Isotónicos: estos ejercicios son los que mantienen el tono muscular mediante el acortamiento y alargamiento que se implican en el movimiento, de la misma manera se dice que son los que obligan al músculo a realizar un movimiento soportando un peso estático constante.

Los ejercicios isotónicos propuestos por el terapeuta para uso en el hogar para la estimulación de la parálisis facial son:

- Mira un punto fijo y parpadear: se realizan 30 sesiones.

- Mirar hacia abajo y cerrar el ojo: se realizan 30 sesiones.

- Elevar y soltar rápidamente la ceja: se realizan 10 sesiones.

- Contractar y soltar rápidamente la nariz: se realizan 10 sesiones.

- Rápidamente boquilla cerrada y abierta: se realizan 10 sesiones.

- Aumento de cejas durante 5 segundos: se realizan 5 sesiones.

- Mantener sonrisa cerrada durante 5 segundos: se realizan 5 sesiones.

- Mantener sonrisa abierta durante 5 segundos: se realiza 5 sesiones.

- Hacer puchero abierto y mantenerlo durante 5 segundos: se realiza 5 sesiones (59), (60), (61).

Si el paciente es consecuente con los ejercicios se verán cambios notorios en su evolución, En este protocolo, también se activan puntos motores y áreas de la cara como una forma de estimulación sensorial.

\section{CONCLUSIONES}

Se puede concluir gracias a esta investigación que la efectividad de la terapia miofuncional en pacientes con parálisis facial de tipo central y periférica en la población infantil y adulta, corrobora la teoría y se soporta de manera muy clara, reportando que para la efectividad de la terapia miofuncional en la parálisis facial de tipo periférica se puede conseguir una reeducación de los músculos faciales a nivel unilateral como bilateral de la parálisis facial periférica mejorando los aspectos fáciles con mejor movilidad de la musculatura facial, sin temblores; así articulando los fonemas correctamente. De la misma manera, se puede concluir que la terapia miofuncional se considera importante en la parálisis facial periférica, por el deterioro del nervio facial Vll par craneal, el cual ayuda a corregir con efectividad la musculatura facial.

Revista Científica Signos Fónicos, 2020, 6 (2): 1-21. ISNN 2422-1716. 
Por otro lado, la terapia miofuncional como tratamiento novedoso, no invasivo carece de un impacto significativo para la eficacia mediante ejercicios de estimulación que garantizan una mejora funcional. Los ejercicios reportados en esta revisión sistemática son de gran importancia puesto que ayudan al aumento y disminución del tono muscular, así mismo al aumento de sensibilidad y movilidad según lo requieran acorde a las necesidades del paciente.

Dentro de los protocolos de rehabilitación existen una serie de recomendaciones que se deben realizar con la mejor disposición posible, reportando la importancia que tiene la colaboración del paciente y de su familia para la ejecución de los ejercicios Miofuncionales en casa para que así, el usuario note mejoría y presente una pronta recuperación.

\section{REFERENCIAS BIBLIOGRÁFICAS}

1. Moya Daza MP. Motricidad Orofacial: Pasado, Presente y Futuro. Int J Med Surg Sci. 2020;1-4.

2.Profesor MS, Chamorro J. Terapia Orofacial y Miofuncional. 2020.

3.Hulley SB, Newman TB, Cumming SR. Cómo empezar: anatomía y fisiología de las investigaciones clínicas. Diseño Investig clínicas. 2011;(March):6-17.

4.Methley AM, Campbell S, Chew-graham C, Mcnally R. Acceso abierto PICO, Picos y la araña: un estudio de comparación de especificidad y sensibilidad en tres herramientas de búsqueda para las revisiones sistemáticas cualitativos. 2014.

5.Hutton B, Catalá F, Moher D. La extensión de la declaración PRISMA para revisiones sistemáticas que incorporan metaanálisis en red: PRISMA- NMA. Med Clin (Barc). 2016;147(6):262-6.

6.Rodríguezz MD, Mangas S, Ortiz MG, Rosete HS, Vales O, Hinojosa R. Parálisis facial periférica. Tratamientos y consideraciones. Arch Neurociencias. 2011;16(3):148-55.

7. López-Contreras, Luísa-Fernanda. Grado De Severidad De Disfonía A Través Del Análisis Acústico Vocal En Docentes De Dos Instituciones De La Estrategia De Atención A La Primera Infancia. Revistas Científica Signos Fónicos. 2019: 5 (1). 1-9, https://doi.org/10.24054/01204211.v1.n1.2019.3976.

8. General J, Otorrinolaringología J, Clínica D, Sociedad S. La teoría total El nuevo despliegue de rehabilitación de parálisis del nervio facial. 2014;86-95.

9.Ilves M, Lylykangas J, Rantanen V, Mäkelä E, Vehkaoja A, Verho J, et al. Facial muscle activations by functional electrical stimulation. Biomed Signal Process Control. 2019; 48: 248-54.

10.Francine M, Aline FS. atención multidisciplinaria del periférico parálisis facial: estudio de caso clínico atención multidisciplinaria de la parálisis Atención multidisciplinar parálisis facial periférica: un estudio de caso. 27(2):253-7.

11.Álvarez C A, Mora N., González R. Parálisis facial periférica: Enfoque desde la medicina física y rehabilitación en Costa Rica. Rev Médica Costa Rica y Centroamérica. 2016;72(615) (académico):24955 .

12. Cáceres-Mansilla, Luz-Amparo. Sindrome de Burnout, Trastornos del Sueño y su asociación con las alteraciones vocales en docentes de primera infancia. Revistas Científica Signos Fónicos. 2019: 5 (1). 10-19, https://doi.org/10.24054/01204211.v1.n1.2019.3977 
Jaimes, Peralta, Rodríguez, Ramírez. Revista Científica Signos Fónicos, 2020, 6 (2): 24-42.

13.Chevalier AM. Rehabilitación de las parálisis faciales centrales y periféricas. EMC - Kinesiterapia Med Física [Internet]. 2003;24(2):1-15.

14.Balaguer R, Casaña S, Suárez MM, Escudero M, Dalmau J. Secuelas de las parálisis faciales periféricas. Acta Otorrinolaringol Esp. 2010;61(2):89-93.

15.TEMAS psicosociales asociadas con la parálisis facial periférica en secuelas FASE: ESTUDIO DE CASO. 2013.

16.Linder TE, Abdelkafy W, Cavero-vanek S. La Dirección de la parálisis del nervio facial periférica: si paresia Versus si Parálisis y fuentes de Ambigüedad en Diseños de estudios. 2010;319-27.

17.Trinh TT, Dorbeau C, Philibert C, Lescanne E. Persistent peripheral facial nerve palsy. Eur Ann Otorhinolaryngol Head Neck Dis. 2019;1-2.

18.Novak CB. Rehabilitation Strategies for Facial Nerve Injuries. Semin Plast Surg. 2004;18(1):47-51.

19.Pereira LM, Obara K, Dias JM, Menacho MO, Lavado EL, Cardoso JR. Facial exercise therapy for facial palsy: Systematic review and meta-analysis. Clin Rehabil. 2011;25(7):649-58.

20.Andino L. Universidad Nacional De Chimborazo (motivación). [Tesis de pregrado]. Universidad Nacional del Chimborazo (Ecuador);2017.

21.Internacional R, Internacional R. Miofuncional Orofacial La terapia del habla en la parálisis facial periférica: un enfoque orofacial miofuncional. 2009.

22.Robinson MW, Baiungo J. Facial Rehabilitation: Evaluation and Treatment Strategies for the Patient with Facial Palsy. Otolaryngol Clin North Am. 2018;51(6):1151-67.

23.Alanazi WL, El-fetoh NMA, Alanazi SL, Alkhidhr MA, Alanazi MA, Alonazi DS, et al. Perfil de la parálisis facial en Arar, norte de Arabia Saudita Wasan. 2017;5596-602

24. González E. efectividad del tratamiento rehabilitador en pacientes con parálisis del nervio facial post- parotidectomía y su impacto en la calidad de vida. [Tesis].2014.

25. Fernández P, Fernández C, Díaz J, Schnake MJ, Guerra C, Díaz F, Muñoz M, Paredes C, Salinas P. Musculatura orofacial Estimulación de la musculatura orofacial en niños con síndrome de down. Revista Virtual. 2013: (13).

26.Henrique I, Balandino S, Lopes TDS, Elisa N, Motta DM, Cortes PC, et al. Facial periférica parálisis campana: update tratamiento. 2012;1(2):40-8.

27.Volk GF, Pantel M, Guntinas-Lichius O. Modern concepts in facial nerve reconstruction. Head Face Med. 2010;6(1):1-11.

28.Pérez RA, Latta MA. El kinesio taping como complemento del tratamiento convencional de la parálisis facial periférica idiopática en pacientes del Patronato Municipal de Amparo Social de Latacunga.2015.

29. Navarro-Criollo; Paola-Lorena. Validación de indicadores de perturbación de Jitter y Shimmer en docentes de primera infancia. Revistas Científica Signos Fónicos. 2019: 5 (1). 20-29, https://doi.org/10.24054/01204211.v1.n1.2019.3978

30.Medicina F De, En I, Parálisis LA, Elisabet A, Archiles Á, Natividad T, et al. Universidad de Valladolid "Revisión de las técnicas de evaluación e valoración de su eficacia" 2019. 
Jaimes, Peralta, Rodríguez, Ramírez. Revista Científica Signos Fónicos, 2020, 6 (2): 24-42.

31.Gil LC.Masaje PROTOCOLO PRÁCTICO PARA EL MASAJE FACIAL MIOFUNCIONAL: una técnica de estimulación de la sensibilidad y la motricidad de la musculatura orofacial. 2012.

32.Navarrete ML, Junyent J, Torrent L. Toxina botulínica y parálisis facial. Nuestra experiencia. Acta Otorrinolaringol Esp. 2010;61(4):277-81.

33. León ME, Benzur D, Alvarez J. Parálisis de Bell, reporte de un caso. Rev Esp Cir Oral y Maxilofac. 2013;35(4):162-6. doi.org/10.1016/j.maxilo.2011.10.009

34.La Touche R, Escalante K, Linares MT, Mesa J. Efectividad del tratamiento de fisioterapia en la parálisis facial periférica. Revisión sistemática. Rev Neurol. 2008;46(12):714-8.

35. Konecny P, Elfmark M, Horak S, Pastucha D, Krobot A, Urbanek K, et al. Central facial paresis and its impact on mimicry, psyche and quality of life in patients after stroke. Biomed Pap. 2014;158(1):1337.

36. Saito H. Acta Oto-Laryngologica Una simple evaluación objetiva y la clasificación de los resultados de la parálisis facial. 2012;6489.

37.Rica C. Rehabilitación Orofacial, Tratamiento Precoz Y Preventivo: Terapiamiofuncional. Rev Científica Odontológica. 2012;8(2):35-8.

38.Bowling NN, Abendroth C, Ahmed A. Peripheral facial nerve palsy. J Clin Neurosci. 2019;69(9):257-60.

39.Payá A, Navarro R, Climent I, Redondo M. Recurrent and alternating peripheral facial palsy in a Rehabilitation department. Rehabilitacion. 2019;53(1):60 4.https://doi.org/10.1016/j.rh.2018.06.001

40.Díaz U, Valdés M, Fernández TR, Calero E, Bienzobas E, Moracén T. Correlations between impairment, psychological distress, disability, and quality of life in peripheral facial palsy. Neurologia. 2019;34(7):423-8.

41.Doménech JI, Tornero J, Cruz Toro P, Ortiz Laredo N, Vega Celiz J, Junyent J, et al. Cirugía reparadora de la parálisis facial mediante colgajo libre microvascularizado de músculo gracilis. Acta Otorrinolaringol Esp. 2014;65(2):69-75.

42.María G, Yoli C, Rita B, González A, Rosa I, Avendaño-sosa GM, et al. Experiencia en el manejo de parálisis facial periférica con vendaje neuromuscular en el Centro de Rehabilitación y Educación Especial de Tabasco. Salud en Tabasco. 2015;21(2-3):71-7.

43. Carvajal-Villamizar,Yuliana-Mercedes. Actitudes de los docentes y estudiantes hacia las personas con discapacidad en una institución pública. Revistas Científica Signos Fónicos. 2019: 5 (1). 30-44, https://doi.org/10.24054/01204211.v1.n1.2019.3979

44.Benítez s., Troncoso O., MoyaF. MMOFP. Manejo Integral De La Parálisis Facial Multidisciplinary Management of Facial Paralysis.Rev Med Clin Condes. 2016;27(1):22-8.

45.Alfonso JI. Uso del vendaje neuromuscular en disfunciones orofasciales: intervención desde Fisioterapia y Fonoaudiología. Mov Científico. 2019;13(1):71-6.

46.Rivkees SA, Carlson LL, Reppert SM. Guanine nucleotide-binding protein regulation of melatonin receptors in lizard brain. Proc Natl Acad Sci U S A. 1989;86(10):3882-6.

47.Lindsay RW, Robinson M, Ther P, Lindsay RW, Robinson M, Hadlock TA. Informe de investigación Rehabilitación integral facial mejora la función en personas con parálisis facial: 
Jaimes, Peralta, Rodríguez, Ramírez. Revista Científica Signos Fónicos, 2020, 6 (2): 24-42.

Massachusetts Eye and Ear En fi rmary. 2015

48.Landingham SW Van, Diels J, Cristo D, Lucarelli J. La terapia física para la parálisis del nervio facial: aplicaciones para el médico. 2018;469-75.

49.Chao JR, Chang J, Lee JH. Extended Epitympanotomy for Facial Nerve Decompression as a Minimally Invasive Approach. J Audiol Otol. 2019;23(4):204-9.

50.Chacon MA, Echternacht SR, Leckenby JI. Outcome measures of facial nerve regeneration: A review of murine model systems. Annals of Anatomy.2020;27(1):151410. Doi.org/10.1016/j.aanat.2019.07.011

51. Martínez-Siza, Diana-Carolina. Creencias de los docentes acerca de la educación inclusiva: un estudio fenomenológico. Revistas Científica Signos Fónicos. 2019: 5 (1). 45-59,

https://doi.org/10.24054/01204211.v1.n1.2019.3980

52.Vincent AG, Bevans SE, Robitschek JM, Groom KL, Herr MW, Hohman MH. Sterno-omohyoid Free Flap for Dual-Vector Dynamic Facial Reanimation. Ann Otol Rhinol Laryngol. 2019.

53 Beleño-Melo; Betsy-Patricia. Calidad de vida en la inclusión educativa de niños y adolescentes con discapacidad intelectual. Revistas Científica Signos Fónicos. 2019: 5 (1). 60-72, https://doi.org/10.24054/01204211.v1.n1.2019.3982

54.Souza I, Dias A, Fontes F, Melo L. Métodos Fisioterapêuticos utilizados no Tratamento da Paralisia Facial Periférica: Uma Revisão. Rev Bras Ciências da Saúde. 2015;19(4):315-20.

55. Wenceslau LGC, Sassi FC, Magnani DM, Andrade CRF. Paralisia facial periférica: Actividad Muscular en Diferentes Momentos de Inicio. CoDAS. 2016;28(1):3-9.

56.Devgan BK, Leach W. Facial nerve paralysis in children. Ear Nose ThrJ. 1977;56(5):41-6.

57.Labbé D, Guerreschi P. Facial paralysis sequelae: Long term follow-up revision surgery, redo, continuing care. Ann Chir Plast Esthet. 2019;64(5-6):531-9. Doi.Org/10.1016/J.Anplas.2019.07.020

58. Gocko X, Poulteau S, Beyens MN, Bertholon P, Pozzetto B. Case report: Recurrent peripheral facial paralysis following two influenza vaccinations in 2009 and 2016. Vaccine.2019;37(35):4864-6. Doi.org/10.1016/j.vaccine.2019.07.025

59.Paschoal R. Evaluación de un protocolo de rehabilitación orofacial en parálisis facial periférica. 2009

60.Herceg M, Frey M. Therapiema física SS participado en la parálisis facial periférica: aplicaciones indicaciones, tiempo y prácticos El tratamiento conservador y Rehabilitación de parálisis facial periférica. 2010;109-14.

61.Bernard C. Kinesiterapia de las parálisis faciales. EMC - Kinesiterapia - Med Física. 2013;34(4):111. 